\title{
Análise da incidência de dengue em pacientes notificados em Montes Claros entre 2017 e 2019
}

RESUMO | Objetivo: analisar a incidência de casos notificados de dengue em Montes Claros. Método: estudo descritivo, exploratório, documental, retrospectivo, transversal, quantitativo, realizado no SINAN online da Secretaria Municipal de Saúde. A amostra foi constituída por 10.879 pacientes notificados entre 2017 e 2019. O tratamento dos dados se deu por epidemiologia descritiva simples. Resultados: a incidência de casos em 2017 foi baixa, quando comparada aos anos subseqüentes. Em 2018, o aumento percentual em relação a 2017 foi de 362,3\%. Em 2019, houve um aumento exponencial na incidência, de modo a atingir $912,8 \%$ em relação a 2018 e 4.592,3\% em relação a 2017. Ainda, houve aumento dos casos confirmados laboratorialmente como positivos em 2019 (3,8\%). Conclusão: a incidência de casos notificados para a doença da dengue mostrou-se alta em 2019 e progressivo com o tempo. O aumento sintomático dos casos mostrou-se bem altos, devendo-se intensificar o acompanhamento desses pacientes.

Palavras-chaves: Dengue. Vírus da Dengue. Notificação de doenças. Incidência. Sistemas de informação em saúde.

\begin{abstract}
Objective: to analyze the incidence of reported cases of dengue in Montes Claros. Method: descriptive, exploratory, documentary, retrospective, transversal study, quantitative, carried out at SINAN online of the Municipal Health Secretariat. The sample consisted of 10,879 patients notified between 2017 and 2019. The data treatment was done by simple descriptive epidemiology. Results: the incidence of cases in 2017 was low when compared to subsequent years. In 2018, the percentage increase compared to 2017 was $362.3 \%$. In 2019, there was an exponential increase in the incidence, to reach $912.8 \%$ in relation to 2018 and $4,592.3 \%$ in relation to 2017. In addition, there was an increase in laboratory confirmed cases as positive in 2019 (3.8\%). Conclusion: the incidence of reported cases of dengue disease was high in 2019 and progressive over time. The symptomatic increase of cases was very high, and the follow-up of these patients should be intensified.
\end{abstract}

Keywords: Dengue. Dengue virus. Disease notification. Incidence. Health information systems.

RESUMEN | Objetivo: analizar la incidencia de los casos reportados de dengue en Montes Claros. Método: estudio descriptivo, exploratorio, documental, retrospectivo, transversal, cuantitativo, realizado en el SINAN en línea de la Secretaría Municipal de Salud. La muestra estaba formada por 10.879 pacientes notificados entre 2017 y 2019. El tratamiento de los datos fue mediante epidemiología descriptiva simple. Resultados: la incidencia de casos en 2017 fue baja en comparación con los años siguientes. En 2018, el incremento porcentual respecto a 2017 fue del 362,3\%. En 2019 se ha producido un aumento exponencial de la incidencia, de forma que se ha alcanzado el $912,8 \%$ respecto a 2018 y el $4.592,3 \%$ respecto a 2017 . Además, hubo un aumento en el número de casos confirmados en los laboratorios como positivos en 2019 (3,8\%). Conclusión: la incidencia de los casos reportados de la enfermedad del dengue fue alta en 2019 y progresiva en el tiempo. El aumento sintomático de los casos fue muy elevado, por lo que se debe intensificar el seguimiento de estos pacientes.

Palabras claves: Dengue. Virus de la dengue. Notificación de enfermedades. Incidencia.

\section{Patrick Leonardo Nogueira da Silva}

Enfermeiro, Mestrando pelo Programa de Pós-Graduação em Cuidados Primários em Saúde da Universidade Estadual de Montes Claros (PPGCPS/UNIMONTES). Montes Claros, Minas Gerais, Brasil.

ORCID: 0000-0003-2399-9526

\section{Ana Clara Rodrigues Marques}

Acadêmica do curso de graduação em Enfermagem pela Universidade Estadual de Montes Claros (UNIMONTES). Montes Claros, Minas Gerais, Brasil.

ORCID: 0000-0002-7340-1572

\section{Kamila Sampaio de Souza}

Enfermeira, Referência Técnica da Vigilância Epidemiológica da Secretaria Municipal de Saúde de Montes Claros (VE/SMS/MOC). Montes Claros, Minas Gerais, Brasil. ORCID: 0000-0002-9951-5690

\section{Maria Suely Fernandes Gusmão}

Enfermeira, Mestranda pelo Programa de Pós-Graduação em Cuidados Primários em Saúde da Universidade Estadual de Montes Claros (PPGCPS/UNIMONTES). Montes Claros, Minas Gerais, Brasil.

ORCID: 0000-0001-9736-8933

\section{Ana Patrícia Fonseca Coelho Galvão}

Enfermeira, Doutoranda pelo Programa de Pós-Graduação em Ciências da Saúde da Faculdade de Ciências Médicas da Santa Casa de São Paulo (PPGCS/FCMSCSP). São Paulo, São Paulo, Brasil.

ORCID: 0000-0003-3376-5678

Recebido em: 07/02/2021

Aprovado em: 04/03/2021

\section{José Ronivon Fonseca}

Enfermeiro, Mestre em Cuidado Primário em Saúde, Professor do Departamento de Enfermagem da Universidade Estadual de Montes Claros (UNIMONTES). Montes Claros, Minas Gerais, Brasil.

ORCID: 0000-0002-4318-7114

INTRODUÇ̃̃O

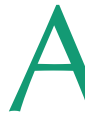
dengue é uma doença infecciosa causada por um vírus, sendo este o Vírus da Dengue ou Dengue Vírus (DENV), constituído por Ribonucleic Acid (RNA), sendo um arbovírus do gênero Flavivirus, da família Flaviviridae, que tem como principal vetor o Aedes aegypti ${ }^{(1)}$. Fatores como a rápida urbanização, o aumento populacional, condições desfavoráveis de saneamento 
e abastecimento de água, o movimento migratório acentuado e a capacidade de adaptação do mosquito ao meio doméstico contribuem para a proliferação do vetor $^{(2)}$. A dengue apresenta-se de forma endêmica em vários países tropicais e subtropicais, a exemplo do Brasil, o que indica que cerca de $40 \%$ da população mundial encontra-se em risco de infectar-se com o vírus ${ }^{(3)}$. No mundo, há a circulação de quatro sorotipos da dengue (DENV-1, DENV-2, DENV-3 e DENV-4) ${ }^{(1)}$. Diante da abrangência geográfica, número de casos e gravidade da doença, esta arbovirose traz consigo implicações de cunho social, econômico e de saúde pública(4). Representa, assim, um desafio à assistência em saúde e à vigilância epidemiológica $(\mathrm{VE})^{(5)}$.

A infecção pelo DENV pode ser sintomática ou assintomática. Em situações em que a dengue é sintomática, a doença apresenta um amplo espectro clínico. Pode variar de casos em que apresentam poucos sintomas a casos graves passíveis de óbito ${ }^{(1)}$. De forma geral, os pacientes evoluem para recuperação após uma dengue auto-limitada, porém debilitante, caracterizada por febre de início abrupto, cefaléia, adinamia, mialgia, artralgia, dor retroorbitária, inapetência e erupção cutânea $^{(4,5,6)}$. Por outro lado, uma pequena parcela evolui para uma forma grave da doença que tem como característica o sangramento, disfunção grave de órgãos ou extravasamento de plasma ${ }^{(4,5)}$. Antes da evolução para a forma grave da dengue, há o surgimento dos sinais de alarme, sendo estes: dor abdominal intensa e contínua; vômitos persistentes; acúmulo de líquidos como ascite; derrame pleural e derrame pericárdico; hipotensão postural e/ou lipotimia; hepatomegalia maior do que 2,0 $\mathrm{cm}$ abaixo do rebordo costal; sangramento de mucosa; letargia e/ou irritabilidade e aumento progressivo do hematócrito ${ }^{(6)}$. Dessa forma, o diagnóstico da dengue é imprescindível para o manejo clínico correto em benefício do paciente para a VE e para a pesquisa ${ }^{(4)}$.

O enfrentamento ocorre desde a simples adoção de medidas de rotina, como as visitas domiciliares (VD), a promoção de forças tarefas e educação em saúde, que visam eliminar os criadouros do vetor e fornecer informações a população sobre a doença, até a elaboração de políticas urbanas que buscam intensificar as ações de combate ${ }^{(7,8)}$. Os Agentes Comunitários de Saúde (ACS) e Agentes de Combate a Endemias (ACE), juntos à população, são os principais responsáveis pela identificação e destruição dos reservatórios de água parada onde os mosquitos se proliferam ${ }^{(9)}$. No Brasil, a dengue é uma doença de notificação compulsória, onde todo caso suspeito e/ou confirmado deve ser informado ao serviço de VE por meio do Sistema de Informação de Agravos de Notificação (SINAN)(5). A VE permite o acompanhamento dos números de casos e sua distribuição territorial, o que possibilita a identificação da demanda por ações de intervenções ${ }^{(7)}$.

No Estado de Minas Gerais, os casos de dengue têm sido registrados desde 1980 e vêm apresentando, ao longo dos anos, um aumento considerável no número de focos de $A$. aegypti ${ }^{(10,11)}$. Nos últimos anos, a cidade de Montes Claros, sendo ela uma das principais do norte mineiro, tem apresentado grande preocupação em relação à dengue. O município que conta com uma população estimada de mais de 409 mil habitantes, com densidade demográfica de 101,41 hab/km2, foi classificado em situação de risco de surto, segundo o Levantamento de Índice Rápido do A. aegypti (LIRAa), por três anos seguidos ${ }^{(11,12)}$.

Nesse contexto, o presente estudo tem como objetivo analisar a incidência de casos de dengue notificados no município de Montes Claros, Minas Gerais, entre 2017 e 2019.

\section{MÉTODO}

Trata-se de um estudo descritivo, exploratório, documental, retrospectivo, transversal, com abordagem quantitativa, realizada na Secretaria Municipal de Saúde de Montes Claros (SMS/MOC), Minas Gerais. Foram utilizadas as fichas de noti- ficação dos pacientes com dengue ao qual foram cadastrados no SINAN. A população do estudo foi constituída por todos os residentes no município o qual atualmente conta com 83 unidades de Estratégia Saúde da Família (ESF) contendo 141 equipes de saúde, seis hospitais e uma Unidade de Pronto-Atendimento (UPA), com cobertura de $100 \%$. Com isso, a amostra foi composta por 10.879 pacientes notificados durante o período de janeiro de 2017 a dezembro de 2019.

Foram elencados os seguintes critérios de elegibilidade para a inclusão na amostra do estudo: (1) residir em Montes Claros; (2) ter sido notificado em uma instituição de saúde do município de Montes Claros; (3) paciente notificado com dengue entre 2017 e 2019; e (4) ter a ficha de notificação cadastrada no SINAN com todos os dados disponíveis no sistema.

Foi enviada uma carta de apresentação e um Termo de Consentimento Institucional ( $\mathrm{TCl}$ ), juntamente com uma cópia do projeto de pesquisa, à Coordenação de VE da SMS/MOC para autorização do estudo. A instituição foi devidamente orientada quanto às diretrizes da pesquisa tendo a SMS/MOC assinado o TCl de modo a autorizar a realização do estudo. A coleta de dados foi realizada no $2^{\circ}$ semestre de 2020, durante os meses de outubro e novembro, pelo pesquisador responsável.

O estudo utilizou dados secundários disponíveis no SINAN online da SMS/ MOC, os quais possibilitam a consulta por município de residência do paciente notificado, cujos quais foram tabulados no TABWIN, do Departamento de Informática do Sistema Único de Saúde (DATASUS), e associados às informações do Centro de Controle de Zoonoses (CCZ). Ressalta-se que o CCZ é responsável pela divulgação do levantamento de número de focos identificados nas regiões do município de Montes Claros por meio do LIRAa. Aplicou-se um formulário semiestruturado de elaboração própria, baseado na ficha de notificação da dengue disponível no site do SINAN, como instrumento de coleta de dados para a captação da amostra. 
As informações coletadas foram divididas em seis variáveis: sexo; faixa etária;

número de casos; realização de exames sorológicos; classificação dos casos con-

\section{Gráfico 1 - Incidência de casos de dengue notificados no município de Montes Claros,} Minas Gerais, nos anos de 2017, 2018 e 2019. Montes Claros (MG), 2020. (n=10.879)

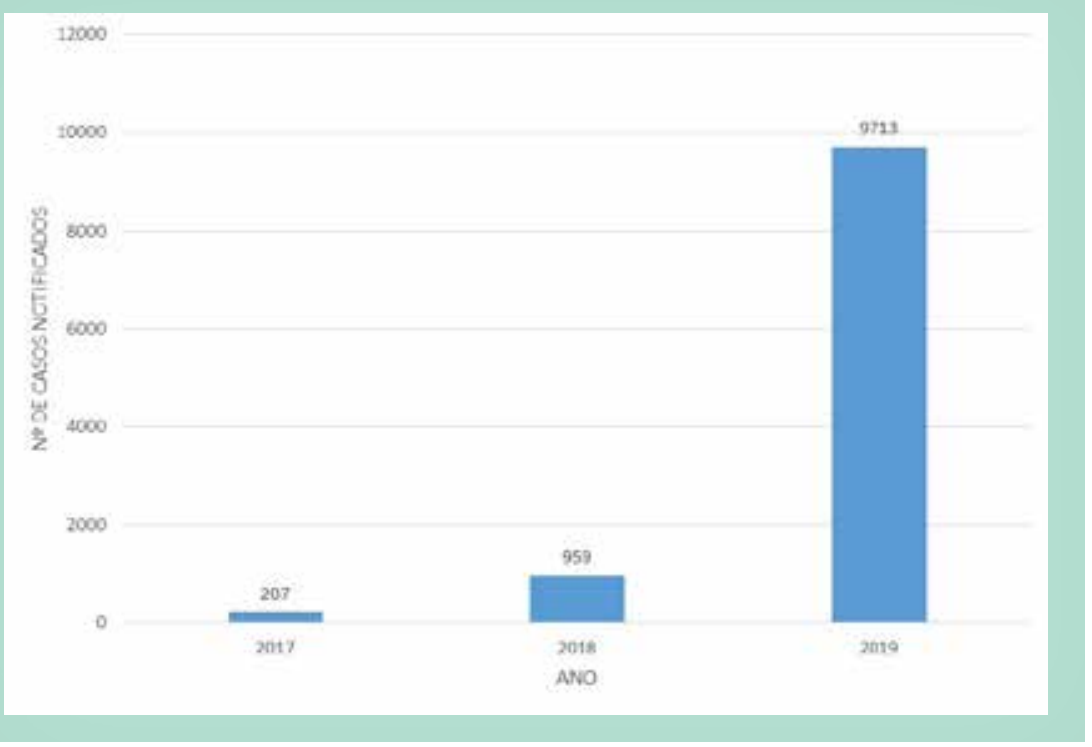

Fonte: SINAN/SMS/MOC, 2020.

Tabela 1 - Incidência de casos notificados de dengue conforme sexo e faixa etária. Montes Claros (MG), 2020. ( $n=10.879)$

\begin{tabular}{lccccccccc}
\multicolumn{1}{r}{ Variáveis } & \multicolumn{2}{c}{2017} & \multicolumn{2}{c}{2018} & \multicolumn{2}{c}{$2019 *$} & \multicolumn{2}{c}{ Total } \\
& $\mathbf{n}$ & $\%$ & $\mathbf{n}$ & $\%$ & $\mathbf{n}$ & $\%$ & $\mathbf{n}$ & $\%$ \\
Sexo & & & & & & & & \\
\hline Masculino & 98 & 0,9 & 389 & 3,57 & 4.040 & 37,14 & 4.527 & 41,61 \\
\hline Feminino & 108 & 1,08 & 570 & 5,23 & 5.673 & 52,06 & 6.351 & 58,37 \\
\hline Ignorado & 01 & 0,02 & 0,0 & 0,0 & 0,0 & 0,0 & 01 & 0,02 \\
\hline Total & 207 & 2,0 & 959 & 8,8 & 9.713 & 89,2 & 10.879 & 100,0
\end{tabular}

Faixa etária (anos)

\begin{tabular}{|lcccccccc|}
\hline$<1$ & 05 & 0,04 & 21 & 0,19 & 226 & 2,12 & 252 & 2,35 \\
\hline $1-4$ & 08 & 0,07 & 43 & 0,39 & 617 & 5,68 & 668 & 6,14 \\
\hline $5-9$ & 04 & 0,03 & 81 & 0,74 & 857 & 7,89 & 942 & 8,66 \\
\hline $10-14$ & 11 & 0,1 & 92 & 0,84 & 1.019 & 9,37 & 1.122 & 10,31 \\
\hline $15-19$ & 26 & 0,23 & 101 & 0,92 & 1.003 & 9,24 & 1.130 & 10,39 \\
\hline $20-34$ & 85 & 0,78 & 294 & 2,7 & 2.849 & 26,2 & 3.228 & 29,68 \\
\hline $35-49$ & 36 & 0,33 & 189 & 1,73 & 1.793 & 16,49 & 2.018 & 18,55 \\
\hline $50-64$ & 24 & 0,22 & 105 & 0,96 & 985 & 9,06 & 1.114 & 10,24 \\
\hline $65-79$ & 08 & 0,2 & 27 & 0,24 & 309 & 2,72 & 344 & 3,16 \\
\hline 80 e + & 00 & 0,0 & 06 & 0,09 & 51 & 0,43 & 57 & 0,52 \\
\hline Total & 207 & 2,0 & 959 & 8,8 & 9.709 & 89,2 & 10.875 & 100,0 \\
\hline
\end{tabular}

Fonte: SINAN/SMS/MOC, 2020. *Em 2019, quatro fichas notificadas não apresentavam informações sobre a faixa etária. forme grau de complexidade; e evolução dos casos. Os dados encontrados foram armazenados no software Statistical Package for the Social Sciences (SPSS), versão 26, e representados por meio de gráficos e tabelas, construídos por meio do Microsoft Excel $\AA^{\circledR}$, Windows for Windows, versão 2010, com freqüências absolutas ( $\mathrm{n})$ e percentuais (\%) para melhor visualização e discussão dos resultados. Os mesmos foram discutidos conforme epidemiologia descritiva simples não-paramétrica e não-probabilística.

O estudo obedeceu aos preceitos éticos estabelecidos pela Resolução $n^{\circ} 466$, de 12 de dezembro de 2012, do Conselho Nacional de Saúde (CNS), o qual regulamenta a realização de pesquisas envolvendo seres humanos ${ }^{(13)}$. O projeto de pesquisa foi apreciado e aprovado pelo Comitê de Ética em Pesquisa da Universidade Estadual de Montes Claros (CEP UNIMONTES) sob parecer consubstanciado $\mathrm{n}^{\circ}$ 4.272.176/2020, Certificado de Apresentação para Apreciação Ética (CAAE) no 37459620.2.0000.5146.

\section{RESULTADOS}

A incidência de casos notificados para a dengue no ano de 2017 foi baixa quando comparada aos anos subseqüentes. Em 2018, o aumento percentual em relação ao ano de 2017 foi de 363,28\%. Em 2019, houve um aumento exponencial na incidência, de modo a atingir 912,82\% em relação ao ano de 2018 e 4.592,27\% em relação ao ano de 2017 (Gráfico 1).

Os dados coletados apontam uma incidência ligeiramente superior para o sexo feminino (58,37\%) nos três anos pesquisados, sendo que em 2019 foi o ano de maior incidência de casos. Quanto à faixa etária, a mais acometida foi a de 20 a 34 anos, com destaque também para o ano de 2019 ( $\mathrm{n}=2.849 ; 29,33 \%$ ). Uma pequena perda de dados foi identificada nos casos por faixa etária desse mesmo ano, sendo registrados 9.709 casos, quando o total notificado foi de 9.713 (Tabela 1). 
Quanto aos casos de dengue analisados por meio dos exames laboratoriais, houve um aumento de 3,76\% de casos testados e confirmados como positivo desde o ano de 2017 ( $n=9 ; 0,08 \%$ ) ao ano de 2019 ( $n=417 ; 3,84 \%)$. A Tabela 2 esque- matiza a representação dos resultados laboratoriais tendo em vista a realização ou não dos exames para a doença. Ressalta-se que uma parcela considerável da amostra foi categorizada como ignorado/branco ou exame laboratorial não realizado.

\begin{tabular}{|c|c|c|c|c|c|c|c|c|}
\hline \multirow{2}{*}{ Resultado } & \multicolumn{2}{|c|}{2017} & \multicolumn{2}{|c|}{2018} & \multicolumn{2}{|c|}{2019} & \multicolumn{2}{|c|}{ Total } \\
\hline & $\mathrm{n}$ & $\%$ & $\mathbf{n}$ & $\%$ & $\mathbf{n}$ & $\%$ & $\mathrm{n}$ & $\%$ \\
\hline Ignorado/Branco & 70 & 0,64 & 574 & 5,27 & 8.683 & 79,82 & 9.327 & 85,73 \\
\hline Positivo & 09 & 0,08 & 237 & 2,17 & 417 & 3,84 & 663 & 6,09 \\
\hline Negativo & 34 & 0,31 & 146 & 1,34 & 612 & 5,63 & 792 & 7,28 \\
\hline Inconclusivo & 01 & 0,02 & 00 & 0,0 & 00 & 0,0 & 01 & 0,02 \\
\hline Não realizado & 93 & 0,95 & 02 & 0,02 & 01 & 0,09 & 96 & 0,88 \\
\hline Total & 207 & 2,0 & 959 & 8,8 & 9.713 & 89,2 & 10.879 & 100,0 \\
\hline
\end{tabular}

Fonte: SINAN/SMS/MOC, 2020

Tabela 3 - Distribuição da incidência de casos de dengue notificados quanto a sua classificação conforme o grau de complexidade. Montes Claros (MG), 2020. ( $n=10.879)$

\begin{tabular}{lccccccccc}
\multicolumn{1}{c}{ Resultado } & \multicolumn{2}{c}{2017} & \multicolumn{2}{c}{2018} & \multicolumn{2}{c}{2019} & \multicolumn{2}{c}{ Total } \\
& $\mathbf{n}$ & $\%$ & $\mathbf{n}$ & $\%$ & $\mathbf{n}$ & $\%$ & $\mathbf{n}$ & $\%$ \\
\hline Descartado & 93 & 0,85 & 718 & 6,6 & 1.326 & 12,21 & 2.137 & 19,66 \\
\hline Dengue clássica & 85 & 0,78 & 232 & 2,14 & 8.352 & 76,76 & 8.669 & 79,68 \\
\hline Dengue com sinais de alarme & 00 & 0,0 & 03 & 0,02 & 30 & 0,28 & 33 & 0,3 \\
\hline Dengue grave & 00 & 0,0 & 03 & 0,02 & 00 & 0,0 & 03 & 0,02 \\
\hline Inconclusivo & 29 & 0,37 & 03 & 0,02 & 05 & 0,05 & 37 & 0,34 \\
\hline Total & 207 & 2,0 & 959 & 8,8 & 9.713 & 89,2 & 10.879 & 100,0
\end{tabular}

Fonte: SINAN/SMS/MOC, 2020.

\begin{tabular}{lccccccccc}
$\begin{array}{l}\text { Tabela } 4 \text { - Prevalência da evolução dos casos de dengue notificados. Montes Claros } \\
\text { (MG), 2020. (n=10.879) }\end{array}$ & \multicolumn{1}{c}{2017} & 2018 & 2019 & \multicolumn{2}{c}{ Total } \\
\multicolumn{1}{c}{ Resultado } & $\mathbf{n}$ & $\%$ & $\mathbf{n}$ & $\%$ & $\mathbf{n}$ & $\%$ & $\mathbf{n}$ & $\%$ \\
& 89 & 0,92 & 951 & 8,74 & 9.696 & 89,02 & 10.736 & 98,68 \\
\hline Cura & 00 & 0,0 & 01 & 0,01 & 00 & 0,0 & 01 & 0,01 \\
\hline Óbito por agravo & 01 & 0,01 & 04 & 0,03 & 03 & 0,02 & 08 & 0,07 \\
\hline Óbito por outras causas & 00 & 0,0 & 00 & 0,0 & 02 & 0,01 & 02 & 0,03 \\
Óbito em investigação & 117 & 1,07 & 03 & 0,02 & 12 & 0,15 & 132 & 1,21 \\
\hline lgnorado/Branco & 207 & 2,0 & 959 & 8,8 & 9.713 & 89,2 & 10.879 & 100,0
\end{tabular}

Fonte: SINAN/SMS/MOC, 2020.

Quanto à classificação dos casos de dengue notificados conforme o seu grau de complexidade, observou-se um aumento significativo de $74,62 \%$ do ano de 2018 para o ano de 2019 com relação à dengue clássica. Ainda, nos anos de 2017 e 2018, prevalecem os casos descartados seguidos dos casos de dengue clássica, enquanto que em 2019, contrapondo-se aos anos anteriores, prevalecem os casos de dengue clássica seguidos dos casos descartados (Tabela 3).

No que diz respeito à evolução dos casos, a cura da doença se deu na maior parte dos casos, tendo uma porcentagem de $89,02 \%$ de cura no ano de 2019 e um aumento percentual de cura de $80,28 \%$ do ano de 2018 para o ano de 2019. O percentual de óbitos pela doença, em comparação a amostra total, não foi significativa $(0,11 \%)$, porém as medidas de promoção e prevenção devem ser intensificadas a fim de se evitar o aumento da mortalidade (Tabela 4).

\section{DISCUSSÃO}

No decorrer dos anos de 2017, 2018 e 2019, houve um aumento progressivo quanto aos casos de dengue na cidade de Montes Claros. O ano de 2019 apresentou um crescimento significativo em relação aos anos anteriores, sendo que dados de outros estudos científicos referentes a Minas Gerais convergem com essa informação ao descreverem que no mesmo ano o Estado apresentou dados alarmantes de casos notificados de dengue de acordo com a proporção de notificações cadastradas no SINAN ${ }^{(14,15)}$.

O clima é um fator relevante para a proliferação do vetor da dengue. A maior parte do Brasil apresenta um clima tropical, quente e úmido, com períodos de oscilação entre chuva e estiagem, que permite a adaptação do A. aegypti ao país e aumenta sua velocidade de reprodução ${ }^{(16)}$. A Microrregião de Montes Claros é composta por 22 municípios e se encontra na Mesorregião norte de Minas Gerais $^{(17)}$. Essa microrregião está em uma 
área de transição entre o clima semi-úmido e o clima semi-árido(18). Com isso, o fator climático da região pode ser um facilitador para a ocorrência das infecções pelo vírus da dengue.

Variáveis socioeconômicas, ambientais e sanitárias também podem ser determinantes na infecção ${ }^{(14)}$. O crescimento e concentração demográfica decorrente do processo de urbanização acelerado e desordenado, aliados à precariedade do saneamento básico, tais como lixões expostos a céu aberto, resíduos sólidos jogados e queimados em terrenos inutilizados e água armazenada incorretamente, são fatores que podem propiciar a disseminação da dengue nas cida$\operatorname{des}^{(18)}$. Segundo o censo de 2020 do Instituto Brasileiro de Geografia e Estatística (IBGE), Montes Claros conta com uma população estimada de 413.487 pessoas, a maior da região Norte de Minas ${ }^{(12)}$. De acordo com dados de 2010 da mesma instituição, 93.4\% dos domicílios apresentam esgotamento sanitário adequa$\mathrm{do}^{(17)}$. Montes Claros é um município de grande porte, o que atribui um papel de disseminador e/ou receptor da dengue. Justamente por ser a cidade mais populosa do norte de Minas Gerais, é referência nos setores de economia, comércio e educação, colaborando para grande circulação de pessoas na cidade. A busca por serviços de saúde qualificados, educação, trabalho e consumo contribui para o movimento das pessoas, que saem das cidades menores para as maiores, de modo que isso pode desempenhar um papel significativo nos eventos de epide$\mathrm{mia}^{(19)}$. São necessários mais estudos que confirmem essa associação(20).

Quanto ao diagnóstico, dos 10.879 casos notificados entre os anos de 2017 e 2019, 663 testaram positivo de acordo com exames laboratoriais. No entanto, apesar dos casos terem sidos confirmados por meio de exame laboratorial, o resultado permite vieses, em virtude do período de coleta da amostra de sangue. Segundo o protocolo do Ministério da Saúde (MS), a sorologia, realizada por meio do método Enzyme-Linked Immunosorbent Assay (ELISA), uma das formas de confirmação laboratorial da dengue, deve ser solicitada a partir do sexto dia do início dos sintomas ${ }^{(6)}$. É possível que se os

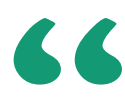

\section{Erros de diagnóstico, dificuldades}

para acessar os serviços de saúde e a ocorrência de infecções assintomáticas são problemas freqüentemente relacionados à subnotificação, a fiel notificação é essencial para que a realidade local seja identificada e o planejamento efetivo de ações em saúde seja realizado pelos gestores sintomas comecem a desaparecer antes do sexto dia os indivíduos deixem de realizar o exame laboratorial, o que reflete nas estatísticas. Entende-se que os demais casos notificados foram confirmados mediante exames clínico-epidemiológicos. Entretanto, deve-se considerar que o quadro clínico da dengue pode ser confundido com os quadros de outras arboviroses como a Zika e a Chikungunya devido às semelhanças, e isso pode interferir nos registros dos diagnósticos ${ }^{(21) .}$

Em relação ao grau de complexidade, constatou-se a dengue clássica na maioria dos casos em comparação à dengue grave e à dengue com sinais de alarme entre os anos de 2017 e 2019. Em contrapartida a outros estudos realizados no município de Barreiras no estado da Bahia, entre 2007 e 2013, e no município de Fortaleza no Estado do Ceará nos anos de 2001, 2006, 2008, 2011 e 2012, Montes Claros apresenta um número maior de casos descartados e/ou inconclusivos do que de dengue clássica, exceto em $2019^{(22,23)}$. Estes resultados podem ter sido influenciados pela falta de orientação e/ou informação da população acerca da importância do diagnóstico. Neste sentido os dados disponíveis podem não refletir a realidade. Erros de diagnóstico, dificuldades para acessar os serviços de saúde e a ocorrência de infecções assintomáticas são problemas freqüentemente relacionados à subnotificação, a fiel notificação é essencial para que a realidade local seja identificada e o planejamento efetivo de ações em saúde seja realizado pelos gestores $^{(14)}$.

Embora a cidade tenha apresentado uma alta incidência de casos de dengue, foi observado que a maioria evoluiu para cura sendo registrado apenas um óbito por agravo em 2018. Uma pesquisa realizada no país observou que a taxa de letalidade da dengue está associada com o número de unidades de atenção primária à saúde, sendo que quanto maior for acesso da população aos profissionais da atenção básica, menor o risco de óbito $^{(24)}$. Esse fato pode ser observado em 
Montes Claros já que a cidade conta atualmente com 82 Estratégias de Saúde da Família, com uma cobertura de 100\% da população. Outra explicação seria o fato de que a maioria dos casos registrados são de dengue clássica, que é auto-limitada ${ }^{(5)}$. Entretanto, também é preciso levar em consideração a não investigação de todos os óbitos, já que em 2017, por exemplo, $1,07 \%$ das causas de morte foram ignoradas.

O sexo feminino foi tido como o mais acometido o que corrobora com outra pesquisa realizada no mesmo município entre os anos de 2007 e $2011^{(25)}$ e com outros estudos de diferentes regiões do país ${ }^{(20,22,26,27)}$. Isso pode ser explicado pelo fato de o A. aegypti possuir circulação intra e peridomiciliar, locais que geralmente são mais freqüentados pelas mulheres ${ }^{(26)}$. Outra possível explicação é o fato delas buscarem por mais assistência médica do que os homens ${ }^{(20)}$, o que contribui para a subnotificação dos casos masculinos.

Estudos semelhantes, realizados em outros lugares, também constataram que a faixa etária mais acometida se concentra entre os 20 e 49 anos, tratando-se de uma população economicamente ativa, inserida no mercado de trabalho ${ }^{(20,22)}$. Os dados também corroboram com uma pesquisa realizada na região das Américas que constatou maior incidência da dengue no Brasil em adultos jovens ${ }^{(28)}$, e ainda com outro estudo realizado no mesmo município(25).

O presente estudo contém limitações. Algumas perdas de dados foram identificadas durante a realização da pesquisa, o que pode ter interferido na análise de algumas variáveis. O Brasil ainda possui a subnotificação dos casos de dengue
66

Nota-se um aumento gradual das notificações de dengue ao longo dos anos, com destaque para 2019 que apresentou um número alarmante de casos em relação aos outros anos. Houve incidência maior de casos notificados em adultos jovens, do sexo feminino, com idade entre 20-34 anos na qual foram os responsáveis pelo maior número de notificações como uma realidade, o preenchimento das fichas de notificação precisa ser meIhorado ${ }^{(29)}$. Além disso, é perceptível a escassez de estudos relacionados à incidência de dengue em território brasileiro que possam servir como comparativo a outras pesquisas. Gestores e profissionais de saúde carecem de dados epidemiológicos válidos e confiáveis que auxiliem na implantação de medidas segundo a real necessidade da população(30).

\section{CONCLUSÃO}

Nota-se um aumento gradual das notificações de dengue ao longo dos anos, com destaque para 2019 que apresentou um número alarmante de casos em relação aos outros anos. Houve incidência maior de casos notificados em adultos jovens, do sexo feminino, com idade entre 20-34 anos na qual foram os responsáveis pelo maior número de notificações. Em 2017 e 2018, a maioria dos casos notificados foi descartada, enquanto em 2019 a prevalência foi de casos de dengue clássica evoluindo para a cura nos dois últimos anos do estudo.

Portanto, é fundamental que a VE realize o acompanhamento detalhado e contínuo da incidência da dengue no município. Em vista disso, o presente estudo apresentou informações relevantes acerca da situação epidemiológica da dengue em Montes Claros, abrindo caminho para que gestores e trabalhadores de saúde realizem uma reavaliação de políticas públicas aliadas a gestão ambiental e interlocução entre as redes intersetoriais de serviços e a sociedade a fim de implantar novas ações de saúde e de saneamento direcionadas à população para o enfrentamento da dengue.

\section{Referências}

1. Ebi KL, Nealon J. Dengue in a changing climate. Environ Res. [Internet]. 2016 [cited 2020 Aug 21];151:115-23. doi: http://doi.org/10.1016/j.envres.2016.07.026

2. França LS, Macedo CMA, Lima JJP, Silva JM, Almeida MB, Vieira SNS. 0 que está errado? Percepção dos agentes comunitários de saúde e endemias sobre o combate ao Aedes aegypti. Enferm. Actual Costa Rica [Internet]. 2020 [cited 2020 Aug 26];38:61-74. doi: http://dx.doi.org/10.15517/revenf.v0i38.37115

3. Silva-Voorham JM, Tami A, Juliana AE, Rodenhuis-Zybert IA, Wilschut JC, 


\section{Referências}

Smit JM. Dengue: a growing risk to travellers to tropical and sub-tropical regions. Ned. Tijdschr. Geneeskd. [Internet]. 2009 [cited 2020 Aug 26];153:A778. Available from: http://www.ncbi.nlm.nih.gov/pubmed/20025792

4. Guzman MG, Harris E. Dengue. Lancet. [Internet]. 2014 [cited 2020 Aug 27];385(9966):453-65. doi: http://doi.org/10.1016/S0140-6736(14)60572-9 5. Brasil. Ministério da Saúde. Secretaria de Vigilância em Saúde. Coordenação-Geral de Desenvolvimento da Epidemiologia em Serviços. Guia de vigilância em saúde: volume único [Internet]. 2. ed. Brasília: MS, 2017 [cited 2020 Aug 26]. 705p. Disponível em: http://portalarquivos.saude.gov.br/images/pdf/2017/outubro/06/Volume-Unico-2017.pdf

6. Brasil. Ministério da Saúde. Secretaria de Vigilância em Saúde. Departamento de Vigilância das Doenças Transmissíveis. Dengue: diagnóstico e manejo clínico - adulto e criança [Internet]. 5. ed. Brasília: MS, 2016 [cited 2020 Aug 21]. 58p. Available from: http://portalarquivos.saude.gov.br/images/pdf/2016/marco/30/dengue-manejo-adulto-crianca-5d.pdf

7. Freitas RM, Rodrigues CS, Almeida MCM. Estratégia intersetorial para o controle da dengue em Belo Horizonte (Minas Gerais), Brasil. Saúde Soc. [Internet]. 2011 [cited 2020 Aug 27];20(3):773-85. doi: http://doi.org/10.1590/ S0104-12902011000300020

8. Alecrim J, Pereira GCA, Castro JM, Alves HNS, Góis RV. Dengue: o desafio das ações de controle sobre o agravo em um município do leste de Minas Gerais. In: Salgado YCS. Patologia das doenças 2 [Internet]. Ponta Grossa (PR): Atena Editora, 2018 [cited 2020 Aug 27]. p. 16-21. Available from: http:/l www.atenaeditora.com.br/wp-content/uploads/2018/11/patologia2.pdf 9. Zara ALSA, Santos SM, Fernandes-Oliveira ES, Carvalho RG, Coelho GE. Estratégias de controle do Aedes aegypti: uma revisão. Epidemiol. Serv. Saúde [Internet]. 2016 [cited 2020 Aug 27];25(2):391-404. doi: http://doi. org/10.5123/s1679-49742016000200017

10. Minas Gerais. Secretaria de Estado de Saúde. Deliberação CIB-SUS/MG n ${ }^{\circ}$ 1.974 , de 28 de outubro de 2014. Aprova o Plano de Contingência Estadual para o enfretamento da dengue e da febre do Chikungunya - 2014/2015. Belo Horizonte: SESMG, 2014 [cited 2020 Aug 26]. Available from: http://www. saude.mg.gov.br/images/documentos/Del $\% 201974 \% 20-\% 20$ Plano $\% 20$ de $\% 20$ contingência $\% 20$ estadual\%20para $\% 200 \% 20$ enfrentamento $\% 20$ da\%20Dengue\%202015.pdf

11. Minas Gerais. Secretaria de Estado de Saúde de Minas Gerais. Subsecretaria de Vigilância em Saúde. Superintendência de Vigilância Epidemiológica. Diretoria de Vigilância de Agravos Transmissíveis. Coordenação das Doenças Transmissíveis pelo Aedes. Boletim epidemiológico das doenças transmitidas pelo Aedes: dengue, chicungunya e zika [Internet]. Belo Horizonte: SESMG 2020 [cited 2020 Aug 26]. Available from: http://www.saude.mg.gov.br/images/noticias e eventos/000_2020/jan_fev_mar/Boletins_Aedes/Boletim $\% 20$ 14-01-2020.pdf

12. Instituto Brasileiro de Geografia e Estatística. Montes Claros, MG: população [Internet]. Rio de Janeiro: IBGE, 2020 [cited 2020 Aug 26]. Available from: http://cidades.ibge.gov.br/brasil/mg/montes-claros/panorama 13. Brasil. Ministério da Saúde. Conselho Nacional de Saúde. Resolução ${ }^{\circ}$ 466, de 12 de dezembro de 2012 [Internet]. Regulamenta a realização de pesquisas envolvendo seres humanos e dá outras providências. Brasília: CNS 2012 [cited 2020 Aug 26]. Available from: http://bvsms.saude.gov.br/bvs/ saudelegis/cns/2013/res0466_12_12 2012.html

14. Mol MPG, Queiroz JTM, Gomes J, Heller L. Gestão adequada de resíduos sólidos como fator de proteção na ocorrência da Dengue. Rev. Panam. Salud Publica [Internet]. 2020 [cited 2020 Aug 21];44:e22. doi: http://doi.org/10. I RPSP.2020.22

15. Pereira JLO, Carvalho JT, Marçal JD, Gomes LVC, Carvalho DS, Ferreira RP. Situação epidemiológica da dengue, Chikungunya e Zika, no Brasil, em Minas Gerais e no Espírito Santo [resumo expandido]. In: Anais do II Simpósio de Enfermagem do UNIFACIG [Internet]. Manhuaçu: UNIFACIG, 2019 [cited 2020 Aug 21]. 2p. Available from: http://pensaracademico.facig.edu.br/index.php/ simposioenfermagem/article/view/1141

16. Peixoto TM, Cerqueira EM, Andrade JN, Coelho MMP. Práticas educativas no controle da dengue: atuação dos agentes de combate às endemias e per- cepção dos moradores. REVISA [Internet]. 2020 [cited 2020 Aug 26];9(2):26270. doi: https://doi.org/10.36239/revisa.v9.n2.p262a270

17. Minas Gerais. Secretaria de Estado de Saúde de Minas Gerais. Meso e microrregiões do IBGE [Internet]. Belo Horizonte: SESMG, 2010 [cited 2020 Aug 21]. Disponível em: https://www.mg.gov.br/sites/default/files/paginas/ arquivos/2016/ligminas_10_2_04_listamesomicro.pdf

18. Silva FG, Magalhães SCM. Correlação entre fatores climáticos, socioambientais e a dengue na microrregião Montes Claros/MG. Cam. Geogr. [Internet]. 2017 [cited 2020 Aug 21];18(61):231-44. doi: https://doi.org/10.14393/ RCG186115

19. Roseghini WFF, Mendonça F, Ceccato P, Fernandes K. Dengue epidemics in Middle-South of Brazil: climate contraints and some social aspects. Rev. Bras. Climatol. [Internet]. 2011 [cited 2020 Aug 21];(9):94-101. Available from: http://revistas.ufpr.br/revistaabclima/article/viewFile/27522/18336

20. Ferreira AC, Chiaravalloti Neto F, Mondini A. Dengue em Araraquara, SP: epidemiologia, clima e infestação por Aedes aegypti. Rev. Saúde Pública [Internet]. 2018 [cited 2020 Aug 27];52:18. doi: https://doi.org/10.11606/s15188787.2018052000414

21. Sobral MFF, Sobral AIGP. Casos de dengue e coleta de lixo urbano: um estudo na cidade do Recife, Brasil. Ciênc. Saúde Colet. [Internet]. 2019 [cited 2020 Aug 26];24(3):1075-82. doi: http://dx.doi.org/10.1590/141381232018243.10702017

22. Oliveira RMAB, Araújo FMC, Cavalcanti LPG. Aspectos entomológicos e epidemiológicos das epidemias de dengue em Fortaleza, Ceará, 2001-2012. Epidemiol. Serv. Saúde [Internet]. 2018 [cited 2020 Aug 27];27(1):e201704414. doi: https://doi.org/10.5123/s1679-49742018000100014

23. Costa IMP, Calado DC. Incidência dos casos de dengue (2007-2013) e distribuição sazonal de culicídeos (2012-2013) em Barreiras, Bahia. Epidemiol. Serv. Saúde [Internet]. 2016 [cited 2020 Aug 29];25(4):735-44. doi: https:// doi.org/10.5123/s1679-49742016000400007

24. Paixão ES, Costa MCN, Rodrigues LC, Rasella D, Cardim LL, Brasileiro Alcione Cunha et al. Trends and factors associated with dengue mortality and fatality in Brazil. Rev. Soc. Bras. Med. Trop. [Internet]. 2015 [cited 2020 Aug 26];48(4):399-405. doi: https://doi.org/10.1590/0037-8682-0145-2015

25. Rodrigues EHR, Viveiros LL, Paixão ALG, Souto KFG, Barbosa TLA, Gomes LMX. Análise epidemiológica das dengues em Montes Claros, Minas Gerais, Brasil. EFDeportes [Internet]. 2012 [Cited 2020 Aug 28];17(175):1-6. Available from: https://www.efdeportes.com/efd175/analise-epidemiologica-das-dengues.htm

26. Scandar SAS, Vieira $P$, Cardoso Junior RP, Silva RA, Papa M, Sallum MAM. Dengue em São José do Rio Preto, Estado de São Paulo, Brasil, 2005: fatores entomológicos, ambientais e socioeconômicos. Bol. Epidemiol. Paul. (Online) [Internet]. 2010 [cited 2020 Aug 26];7(81):4-16. Available from: http://periodicos.ses.sp.bvs.br/scielo.php?script=sci_arttext\&pid=S1806-42722010000900001\&lng=pt

27. Andrioli DC, Busato MA, Lutinski JA. Características da epidemia de dengue em Pinhalzinho, Santa Catarina, 2015-2016. Epidemiol. Serv. Saúde [Internet]. 2020 [cited 2020 Aug 27];29(4):e2020057. doi: https://doi. org/10.5123/s1679-49742020000400007

28. Martín JLS, Brathwaite O, Zambrano B, Solórzano JO, Bouckenooghe A, Dayan GH et al. The epidemiology of dengue in the americas over the last three decades: a worrisome reality. The American journal of tropical medicine and hygiene [Internet]. 2010 [cited 2020 Aug 26];82(1):128-35. doi: https:// doi.org/10.4269/ajtmh.2010.09-0346

29. Pinto RC, Castro DB, Albuquerque BC, Sampaio VS, Passos RA, Costa CF et al. Fatores preditores de mortalidade em pacientes com dengue grave no Estado do Amazonas, Brasil. Plos One [Internet]. 2016 [cited 2020 Aug 26];11(8):e0161884. doi: https://doi.org/10.1371/journal.pone.0161884 30. Martins AMEBL, Fonseca JR, Moura RSD, Gusmão MSF, Neves PCV, Ribeiro LG et al. Violência contra a mulher em tempos de pandemia da COVID-19 no Brasil. Rev. Enferm. Atual INDERME [Internet]. 2020 [cited 2020 Aug 29];93(esp):e020009. doi: https://doi.org/10.31011/reaid-2020-v.93-n.0art.828 